\title{
Predicting outcome in severe ulcerative colitis
}

\author{
S P L Travis, J M Farrant, C Ricketts, D J Nolan, N M Mortensen, M G W Kettlewell, \\ D P Jewell
}

Treatment with intravenous corticosteroids and a policy of early colectomy originally reduced the mortality in severe episodes of ulcerative colitis from $31-61 \%$ in the $1950 \mathrm{~s}^{12}$ to $5-9 \%$ in $1962 .^{23}$ Although mortality outside specialist centres in 1974 remained alarmingly high $(37 \%, 4)$, in specialist or district hospitals with an interest in colitis it is now 3\% or less, including operative mortality. ${ }^{5-7}$ These figures are pertinent because the introduction of further medical treatment such as cyclosporine $^{8}$ runs the risk of delaying colectomy inappropriately. It remains difficult, however, to predict at an early stage which patients with severe ulcerative colitis will respond poorly to intensive medical treatment and require colectomy.

There is a need for simple clinical and laboratory criteria that will predict outcome and assist the decision to operate on patients with severe ulcerative colitis. A large retrospective study in 1975 identified a persistent tachycardia, fever, hypoalbuminaemia, and radiological features (mucosal islands or dilation) after 24 hour treatment in hospital as being associated with colectomy on that admission. ${ }^{9} \mathrm{~A}$ rising or persistently raised $\mathrm{C}$ reactive protein (CRP) was associated with urgent colectomy in six of eight patients, ${ }^{10}$ but it was not clear whether more frequent measurements of CRP would be of predictive value. It has since been reported that first episodes, extensive disease, ${ }^{6} 11$ and three or more distended loops of small bowel on the initial plain abdominal radiograph ${ }^{12}$ are associated with a poor response to intravenous therapy.

To assess whether the early pattern of change in inflammatory markers or other variables could predict the need for surgery, 36 clinical, laboratory, and radiographic variables have been measured prospectively in patients with severe colitis. The study was performed with the option of using cyclosporine in patients not responding to intravenous corticosteroids and also examined the outcome during a one year follow up period.

\section{Methods}

PATIENTS

All patients with severe ulcerative colitis admitted to the John Radcliffe Hospital between March 1992 and September 1993 were evaluated prospectively. The diagnosis of ulcerative colitis was made on normal clinical, radiological, and pathological criteria and a severe episode defined as the passage of $\geqslant 6$ bloody stools daily with one or more of the following criteria: temperature $>37 \cdot 8^{\circ} \mathrm{C}$, pulse 
$>90 / \mathrm{min}$, haemoglobin $<10.5 \mathrm{~g} / \mathrm{dl}$, or erythrocyte sedimentation rate $(\mathrm{ESR})>30 \mathrm{~mm} / \mathrm{h} .{ }^{3}{ }^{13}$

MEASUREMENTS

In addition to the sex and date of birth of the patient, the following data were collected:

Clinical details - date of diagnosis, duration of current relapse, duration of previous remission, current maintenance therapy, number and timing of previous admissions, maximum extent of macroscopic disease, number of motions in the day prior to admission, abdominal tenderness on palpation, and rigid sigmoidoscopic appearances (loss of vascular pattern, contact bleeding, or ulceration indicated by adherent mucosal slough) on admission.

Clinical observation - daily stool frequency, consistency (unformed, semi-formed, formed), amount of blood (visible, occult blood positive, none), pulse rate, and temperature (both measured six hourly).

Laboratory investigations - blood was taken daily for five days, then as indicated for standard laboratory measurement of full blood count, ESR, CRP, potassium, creatinine, and albumin. In addition, serum was collected daily and stored at $-20^{\circ} \mathrm{C}$ for orosomucoid analysis by nephelometry (Beckman Array, High Wycombe, Bucks). Plain abdominal radiographs were taken on admission and after two days. At the end of the study these were reported by an experienced radiologist unaware of the outcome, for the distribution of faeces (none, up to the hepatic flexure, splenic flexure, or sigmoid colon), presence of mucosal islands, colonic dilatation $>5.5 \mathrm{~cm}$, $\geqslant 3$ distended loops of small bowel gas, ${ }^{12}$ or perforation.

Outcome - Complete response to intensive medical therapy was defined as a stool frequency $\leqslant 3 /$ day on day 7 , with no visible blood in the motions. Incomplete responders were defined as those with a stool frequency $>3$ or visible blood on day 7 who did not require colectomy on that admission. Indications for colectomy were failure to respond or frank deterioration during the first few days of intensive medical therapy; continued diarrhoea, abdominal tenderness or a low grade fever after intensive medical therapy; and perforation, increasing colonic dilatation, or massive haemorrhage. ${ }^{14}$

\section{MANAGEMENT}

All patients received standard intensive medical therapy for severe colitis. ${ }^{15}$ Fluid, electrolyte, and haemoglobin deficiencies were corrected and hydrocortisone $100 \mathrm{mg}$ given intravenously six hourly, with rectal hydrocortisone $100 \mathrm{mg}$ twice daily. This was continued for five to seven days with oral fluids until it was clear that the patient had responded or colectomy was needed. Parenteral nutrition was given to malnourished patients. Incomplete responders were treated with intravenous cyclosporine $4 \mathrm{mg} / \mathrm{kg} /$ day or further intravenous corticosteroids for up to six days, then converted to oral therapy (cyclosporine $5 \mathrm{mg} / \mathrm{kg} /$ day and oral corticosteroids), or referred for colectomy.

STATISTICAL ANALYSIS

Student's unpaired $t$ test was used to compare data on admission. Both the number of episodes (51) and the number of patients (49) were analysed. All 51 episodes were analysed first, then the 49 patients excluding the initial episodes in the two patients who were entered twice and finally the 49 patients excluding the second episodes in these two patients. Repeated measures analysis of variance were used to assess differences between outcomes and to identify potential trends in data during admission in mean bowel frequency, pulse rate, haemoglobin, platelet count, ESR, CRP, orosomucoid, and albumin, using the StatGraphics statistical software package. The analyses were performed initially for five days and then for eight days after admission. As $97 \%$ of all potential data was collected, algorithms that allowed for occasional missing values were used, rather than exclude patients with missing data. Because the analysis was repeated for all eight measurements, the significance level was set at 0.00625 to correct for multiple comparisons $(p=0 \cdot 05 / 8$, Bonferroni's correction). A classification tree ${ }^{16}$ was then developed to try to predict patient outcome from the measures obtained on the third day of treatment.

\section{Results}

PATIENTS

Fifty one episodes were treated in 49 patients (26 male, age 21-77, median 43). Twenty one (42\%) responded completely, 15 (29\%) required colectomy on that admission, and 15 had an incomplete response. Analysing by patients rather than episodes changes the figures by $<2 \%$. Excluding the initial episodes, 21 patients had a complete response, 14 required colectomy, and 14 had an incomplete response. Excluding subsequent episodes, the numbers are 21,13 , and 15 . Two patients had been transferred for treatment from other hospitals, neither of whom required colectomy. Two patients were subsequently found to have Crohn's disease, one at the time of surgery and another after an ileoanal pouch had been formed.

\section{ANTECEDENT DATA}

A third of episodes involved a first episode of colitis, defined as the first episode of symptoms leading to diagnosis, more commonly in complete responders (Table I). For those with a first episode (16 of 49 patients), $12(75 \%)$ responded completely, and three (19\%) required colectomy during the initial admission. For those with a previous episode of colitis, the median duration of remission tended to be shorter in those who required colectomy, as did the time since any previous admission, but the differences did not reach statistical significance. The duration of relapse 
did not differ significantly. Similar proportions of patients were taking maintenance therapy in each group, but numbers are too small to identify any relation between outcome and type of salicylate therapy.

\section{ADMISSION DATA}

The number of Truelove and Witts criteria ${ }^{13}$ on admission in addition to a bloody stool frequency $\geqslant 6 /$ day was similar in those who responded to medical treatment or who required colectomy (Table II). One person who had a colectomy had no additional criteria on admission (and therefore technically only fulfilled the criteria for a moderate episode), but is included because she deteriorated during treatment to meet these criteria. On admission,

TABLE I Patient data prior to admission

\begin{tabular}{lllll}
\hline & Responders & Incomplete & Colectomy & Overall \\
\hline Number of episodes & 21 & 15 & 15 & 51 \\
Age (SD) (y) & $46 \cdot 7(19 \cdot 2)$ & $47 \cdot 5(12 \cdot 3)$ & $43 \cdot 2(15 \cdot 3)$ & $45 \cdot 9(15 \cdot 3)$ \\
First episode (\%) & 57 & 7 & 20 & 31 \\
Previous remission (range, months) & $16(5-38)$ & $15(5-240)$ & $9(3-54)$ & $13(3-240)$ \\
Previous admission (\%) & 56 & 43 & 50 & 49 \\
Time since last admission (range, months) & $67(24-185)$ & $61(8-240)$ & $5(1-103)$ & $47(1-240)$ \\
Salicylate therapy (\%) & 89 & 93 & 83 & 89 \\
Sulphasalazine (\%) & 75 & 23 & 60 & 48 \\
Mesalazine (\%) & 25 & 31 & 10 & 23 \\
Olsalazine (\%) & 0 & 46 & 30 & 29 \\
\hline
\end{tabular}

First episode: \% patients in each group presenting with a first episode of colitis; previous remission: median duration of remission in months before current relapse; previous admission: $\%$ patients previously admitted for treatment, excluding those with first episodes $(n=17$ of 35 ); time since last admission: median time in months in these patients; salicylate therapy: maintenance treatment taken by patients before admission.

TABLE II Patient admission details

\begin{tabular}{lcccc}
\hline & Responders & Incomplete & Colectomy & Overall \\
\hline Number of episodes & 21 & 15 & 15 & 51 \\
Motions/day & $8(2)$ & $8(2)$ & $8(3)$ & $8(2)$ \\
Pulse rate & $106(15)$ & $96(11)$ & $101(14)$ & $101(14)$ \\
Temperature & $37 \cdot 7(0 \cdot 7)$ & $37 \cdot 3(0 \cdot 8)$ & $37 \cdot 6(0 \cdot 4)$ & $37 \cdot 5(0 \cdot 7)$ \\
Haemoglobin (g/dl) & $12 \cdot 6(2 \cdot 6)$ & $11 \cdot 3(2 \cdot 4)$ & $11 \cdot 2(2 \cdot 0)$ & $11 \cdot 8(2 \cdot 4)$ \\
ESR (mm/h) & $41(25)$ & $48(20)$ & $47(28)$ & $45(24)$ \\
CRP (mg/l) & $43(38)^{\star}$ & $89(85)$ & $116(102)$ & $78(81)$ \\
Orosomucoids (mg/dl) & $117(41)$ & $144(55)$ & $158(50)$ & $137(50)$ \\
Truelove and Witts criteria & $2 \cdot 2(1 \cdot 0)$ & $2 \cdot 1(0 \cdot 8)$ & $2 \cdot 1(1 \cdot 3)$ & $2 \cdot 2(1 \cdot 0)$ \\
Extent of disease (\%) & 24 & 20 & & \\
$\quad$ Distal & 19 & 13 & 0 & 16 \\
Left sided & 38 & 13 & 20 & 25 \\
Extensive & 19 & 54 & 60 & 41 \\
Pancolitis & & & & \\
\hline
\end{tabular}

Figures are mean (SD). *Significantly different from the colectomy group $(p=0.005)$. No other differences are significant when corrected for multiple comparisons $(\mathrm{p}<0.00625)$. The mean number of Truelove and Witts criteria are those in addition to bloody stool frequency $\geqslant 6 /$ day. When analysed by number of patients, the means change by $<5 \%$ except for the CRP in incomplete responders, which was $100(86) \mathrm{mg} / \mathrm{l}$ if initial episodes in two patients entered twice were excluded.

TABLE III Analysis of serial data over five days: significance values comparing patients who required colectomy with those who did not

\begin{tabular}{|c|c|c|c|c|c|c|}
\hline & \multicolumn{2}{|c|}{ Episodes $(n=51)$} & \multicolumn{2}{|c|}{$\begin{array}{l}\text { Patients ( } n=49) \\
\text { Excluding first admission }\end{array}$} & \multicolumn{2}{|c|}{$\begin{array}{l}\text { Patients ( } n=49) \\
\text { Excluding second admission }\end{array}$} \\
\hline & Mean & $\Delta$ time & Mean & $\Delta$ time & Mean & $\Delta$ time \\
\hline $\begin{array}{l}\text { Bowel frequency } \\
\text { Pulse rate } \\
\text { Haemoglobin } \\
\text { Platelet count } \\
\text { ESR } \\
\text { CRP } \\
\text { Orosomucoids } \\
\text { Albumin }\end{array}$ & $\begin{array}{r}<0.001 \\
0.012 \\
0.041 \\
0.042 \\
0.119 \\
0.001 \\
0.011 \\
0.035\end{array}$ & $\begin{array}{r}<0.001 \\
0.064 \\
0.947 \\
0.796 \\
0.017 \\
0.025 \\
0.904 \\
0.726\end{array}$ & $\begin{array}{r}<0.001 \\
0.010 \\
0.045 \\
0.056 \\
0.155 \\
0.002 \\
0.013 \\
0.041\end{array}$ & $\begin{array}{r}<0.001 \\
0.026 \\
0.967 \\
0.778 \\
0.013 \\
0.044 \\
0.902 \\
0.714\end{array}$ & $\begin{array}{r}<0.001 \\
0.022 \\
0.076 \\
0.054 \\
0.155 \\
<0.001 \\
0.011 \\
0.029\end{array}$ & $\begin{array}{r}<0.001 \\
0.077 \\
0.965 \\
0.882 \\
0.013 \\
0.018 \\
0.761 \\
0.837\end{array}$ \\
\hline
\end{tabular}

Significance values of the data are presented graphically (see Figure). Data have been analysed by episode and per patient, excluding either the first or second admission of two patients admitted twice. Mean: compares the means between those who required colectomy and those who did not. $\Delta$ time: compares the rate of change over time between the two groups. Over the five day period, stool frequency was significantly higher and decreased more slowly in those who required colectomy; the CRP was significantly higher in the colectomy group, but the rate of change did not differ when corrected for multiple comparisons $(p<0 \cdot 00625$, see text). the presence of severe rectal inflammation on sigmoidoscopy causing the appearance of ulceration was significantly more common $(93 \%)$ in those who required colectomy than those who only had contact bleeding or a granular rectal mucosa $(39 \%, p=0.002)$. The distribution of disease did not differ significantly between groups, although pancolitis was present in $60 \%$ who required colectomy and only in $19 \%$ of complete responders. The initial CRP was significantly higher in the colectomy group compared with those who responded completely (Table II). These data are consistent with the CRP being the most sensitive maker of colonic inflammation.

\section{MANAGEMENT}

Intravenous and rectal hydrocortisone were given for five days only in all patients who responded completely, a median of six days (range 5-8) in incomplete responders and a median of five days (range 2-8) in those who had a colectomy. In 30 of 51 episodes which did not completely respond to hydrocortisone, four had deteriorated sufficiently by five days to need urgent colectomy. Another 12 continued with hydrocortisone for up to three more days until it was clear whether improvement continued (8) or colectomy (4) was needed, and the remaining 14 received intravenous cyclosporine ( $4 \mathrm{mg} / \mathrm{kg} /$ day) for a median four days (range 1-6). In those who received cyclosporine, seven of 14 did not improve and proceeded to colectomy during that admission. The remaining seven of 14 were given oral cyclosporine $(5 \mathrm{mg} / \mathrm{kg} /$ day $)$ when symptoms were under sufficient control to leave hospital, but three required colectomy within three months. Of the incomplete responders, the median stool frequency on discharge was three (range 2-9), with visible blood in $60 \%$ and after a median nine days in hospital (range 7-20). Of the 15 patients who had a colectomy on the same admission, 12 had a subtotal colectomy, one a proctocolectomy, one a colectomy and mucous fistula, and the one found to have Crohn's colitis at operation had a split ileostomy.

PATTERN OF CHANGE DURING FIRST FIVE DAYS Whether analysed by episode or by patient numbers, repeated measures analysis of variance over the first five days showed that the bowel frequency and CRP were significantly higher $(p<0.00625)$ in patients who required colectomy than in those responding partly or completely. The bowel frequency also declined significantly more slowly in the colectomy group (Table III, Figure). At a less rigorous $5 \%$ level of significance, the mean pulse rate, haemoglobin, platelet count, serum albumin, and orosomucoids also differed between those who required colectomy and those who did not. The rate of change in pulse rate, CRP, and ESR was slower in the colectomy group when analysed by patients rather than episodes (Table III). When data were analysed over eight rather than five days, the bowel 
frequency, pulse rate, and CRP were significantly higher $(p<0.00625)$ in those who required surgery. Follow up analysis showed that incomplete responders were more similar to the colectomy group than the complete responders for bowel frequency, but similar to complete responders for the change in CRP.

Two days after admission, the presence of mucosal islands on the plain abdominal
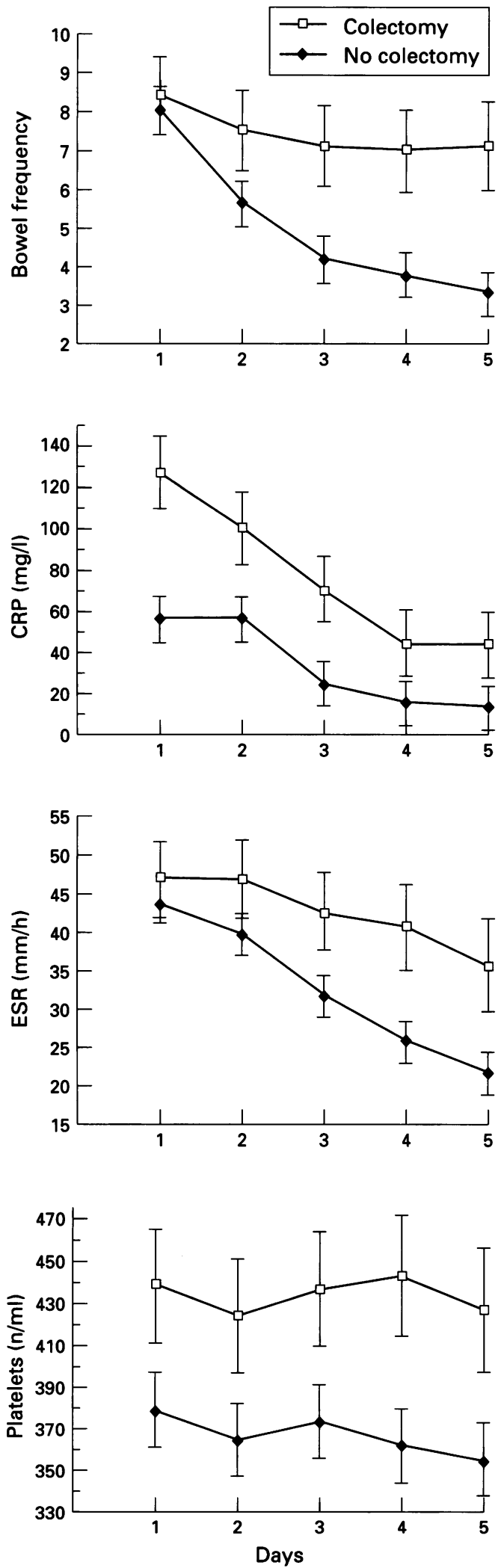

radiograph was significantly more common in those requiring colectomy $(50 \%)$ than those who did not $(10 \%, p=0.013)$. The extent of colitis (pancolitis, extensive, left sided, distal, proctitis) on the admission radiograph agreed within one colonic segment to that identified by barium enema, colonoscopy, or operation in $74 \%$. The plain radiograph overestimated the extent of disease by two or more colonic
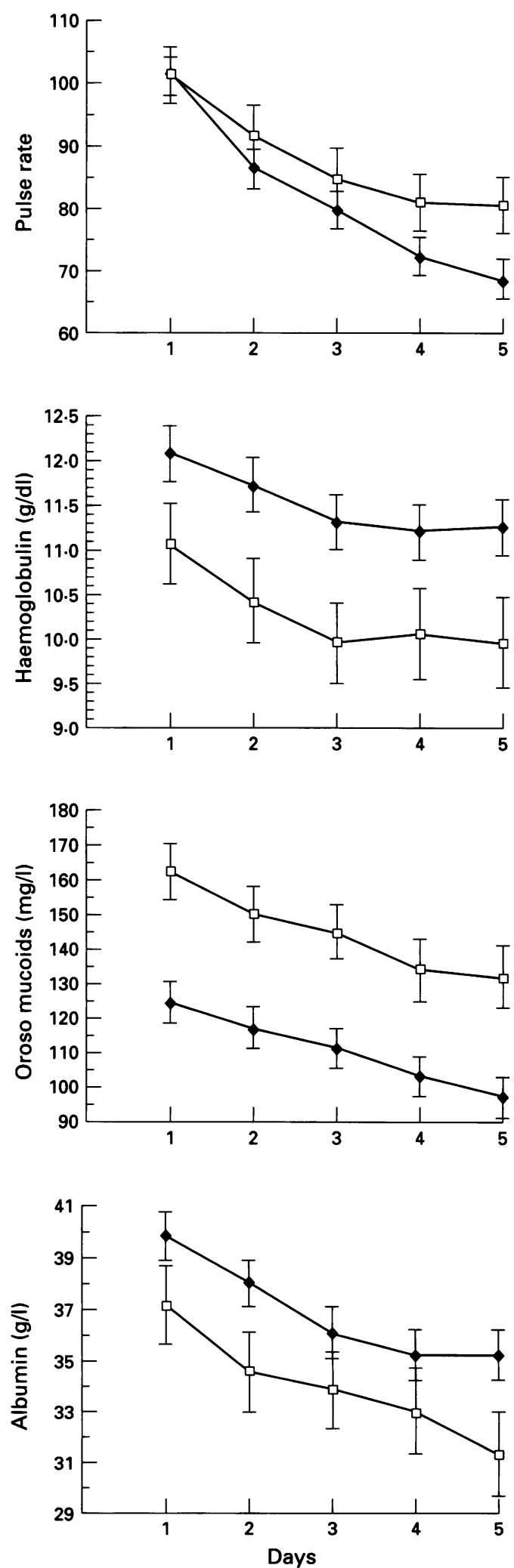

Pattern of change (mean (95\% confidence limits)) for different variables in severe ulcerative colitis during the first five days of treatment (see Table III for significance values). 
segments in $18 \%$ and underestimated the extent in $8 \%$.

PREDICTING OUTCOME ON DAY 3

The simplest rule predicted with $85 \%$ success that patients with more than eight bowel actions on day 3 , or with three to eight bowel actions and a CRP $>45 \mathrm{mg} / \mathrm{l}$ would need colectomy on the same admission. Of those misclassified by this rule, four patients who would have been classified as surgical cases did not undergo colectomy on that admission, but required colectomy in the following months. Three patients underwent colectomy when this classification rule suggested that they should not.

\section{FOLLOW UP DATA}

Patients were followed up for a median period of 12 months (range 3.5-21). For those who had responded completely, none had continuous symptoms and the duration of remission was significantly longer than in incomplete responders $(p<0.001$, Table IV). Immunosuppression with prednisolone, azathioprine or cyclosporine was still necessary at the time of last follow up in $14 \%$ of complete responders, compared with $82 \%$ of incomplete responders. Among the complete responders, colectomy was subsequently needed in one patient $(5 \%)$, compared with six of 15 episodes $(40 \%)$ in incomplete responders. Of those who had a colectomy, 10 of $15(67 \%)$ went on to have an ileoanal pouch (including one which later had to be excised for Crohn's disease) and three of $15(20 \%)$ had a proctectomy after continuous symptoms from the rectal stump. One patient died from a myocardial infarction five months after colectomy.

\section{Discussion}

This study confirms that patients with severe colitis defined by the Truelove and Witts criteria $^{13}$ have a $29 \%$ chance of colectomy on the same admission. ${ }^{514}$ This reflects the inadequacies of medical treatment even with cyclosporine and the prospective data show how difficult it is to predict who will require colectomy. Incomplete responders are shown

TABLE IV Follow up data on patients responding completely or incompletely to medical treatment (median 12 months, range $3 \cdot 5-21$ )

\begin{tabular}{lcc}
\hline & Responders & Incomplete \\
\hline Number of episodes & 21 & 15 \\
Remission (range, months) & $9(2-21)$ & $0(0-12)^{\star}$ \\
Continuous symptoms (\%) & 0 & 60 \\
Relapse (\%) & 43 & 86 \\
Number of relapses (range) & $0(0-4)$ & $1(0-2)$ \\
Readmission (\%) & 36 & 50 \\
Colectomy (\%) & 5 & 40 \\
Immunosuppressants (n) & $3 / 20$ & $9 / 11$ \\
Prednisolone & 2 & 8 \\
Azathioprine & 1 & 4 \\
Cyclosporine & 0 & 3 \\
\hline
\end{tabular}

Remission: median duration of remission, ${ }^{\star} \mathrm{p}<0.001$; relapse: $\%$ relapses in those entering remission; readmission: \% $\%$ relapses in those entering remission; readmission: \% patients readmitted for treatment, including colectomy; time of last follow up; five of nine incomplete responders were taking more than one immunosuppressant. to have a very high risk of continuing symptoms or colectomy in the months after a severe episode.

While the criteria for a complete response were stringent (stool frequency $\leqslant 3$ without visible blood after seven days), this is a reasonable definition of remission for patients suffering the symptoms. Only $42 \%$ of episodes responded completely to intensive medical treatment. By the time that incomplete responders were discharged, $73 \%$ still had a stool frequency $>3 /$ day or visible bleeding. Of the 49 patients, $43 \%$ ultimately required colectomy. Although patients are not normally entered into a study more than once, we feel that analysis of the number of episodes rather than the number of patients is clinically most relevant. This is because a previous severe episode of colitis influences surgical decision making and the principal end point of the study was surgery on that admission. As it happens, analysis by episodes or patients (Table III) made little difference.

There is thus an urgent need for more effective medical treatment. Cyclosporine was ineffective in seven of 14 patients who had not responded to intravenous corticosteroids, and in the $50 \%$ who showed some response to cyclosporine, only four subsequently avoided colectomy and two of these continued to have symptoms. This means that only two of 14 patients $(14 \%)$ can be considered to have entered remission and these two were still receiving prednisolone at the time of follow up. These figures are not encouraging and are in contrast with those described by Present. ${ }^{8}$ It remains possible, however, that earlier use of cyclosporine might be of greater benefit. There is also a potential benefit from partial remission, in that it may allow a patient time to come to terms with the prospect of surgery, especially if the extent of disease is limited.

A severe relapse with a defined course of action (admission for intensive medical therapy) can be identified by a single Truelove and Witts' criterion ${ }^{13}$ in addition to a bloody stool frequency $\geqslant 6 /$ day. Table II shows that there was no difference in the number of criteria on admission between responders, incomplete responders, or those who required colectomy. Objective assessment of relapse in ulcerative colitis is essential if the severity is not to be underestimated, but there seems little need to differentiate between 'severe' and 'fulminant' colitis. Several of those who met all five criteria responded completely to medical therapy and one patient who only had a moderate episode subsequently deteriorated to need urgent colectomy. The two patients who turned out to have Crohn's colitis are included because this reflects clinical practice. Excluding these two did not change the significant variables in the repeated measures analysis of variance.

Only bowel frequency and CRP differed significantly between those responding to medical treatment and those requiring colectomy at the most rigorous level of significance (Table III). The orosomucoids tended to be higher on admission and the ESR to decline more slowly in the colectomy group, which 
favours the CRP as the most useful inflammatory marker. In an attempt to find a more specific marker of colonic inflammation, the serum concentration of nitric oxide metabolites was measured in some patients. These metabolites decreased in a similar fashion to the CRP, but did not discriminate between the groups. ${ }^{17}$ The pulse rate tended to be higher and declined more slowly in those who required colectomy, while the haemoglobin and albumin were lower. These simple measures help in the overall assessment of colitic patients. Surprisingly, the platelet count, temperature, or amount of small bowel gas did not consistently discriminate between those who required colectomy and those who did not.

It might be argued that there is circular reasoning on the premise that we have defined criteria for colectomy and then evaluated the measures that define that decision. Indeed, it has been suggested that the threshold for colectomy in severe ulcerative colitis in Oxford is lower than elsewhere. If this is the case, then published experience supports the practice. ${ }^{356}$ By examining the change in pattern of commonly measured variables, we have tried to predict which patients make a poor response to medical treatment. In Oxford, such patients generally have a colectomy because delaying surgery increases the risk of complications, or of persistent symptoms if medical treatment is continued. No single factor (such as diarrhoea) influenced the surgical decision. All decisions considered the duration, extent and previous pattern of disease, the influence of symptoms on the patient's lifestyle, the inclination of the patient, and response to or side effects from medical treatment.

The follow up data show that there is good reason to be optimistic in those who respond completely ( $5 \%$ colectomy rate, $85 \%$ in remission without immunosuppressants), but every reason to be cautious in other patients. For incomplete responders, $40 \%$ required colectomy within a few months (range 3-30 weeks) and only two of $14(14 \%)$ remained in remission without immunosuppressive therapy. These data are similar to previous reports. ${ }^{35}$ Furthermore, in five patients who had an emergency sub-total colectomy and were not candidates for an ileoanal pouch, four had to have a proctectomy for continuing symptoms. Even though emergency proctocolectomy has been shown to be a safe operation in experienced hands, ${ }^{5}$ a sub-total colectomy was initially performed in these patients because of constraints on theatre time.

What conclusions, then, can be drawn to give objective advice on the management of patients with a relapse of ulcerative colitis? Firstly, the severity of relapse should be assessed objectively. All patients with a bloody stool frequency $>6 /$ day with any additional feature (pulse $>90$, temperature $>37 \cdot 8^{\circ} \mathrm{C}$, haemoglobin $<10.5 \mathrm{~g} / \mathrm{dl}, \mathrm{ESR}>30$ ) should be admitted for intensive treatment. Stool frequency, six hourly pulse rate, and CRP should be monitored daily as a slower rate of improvement in these variables distinguished those who required urgent colectomy from those who did not. Although one must be wary of placing values on individual variables, it is possible with reasonable confidence to predict the outcome on day 3. Patients who continue to have frequent stools ( $>8$ on day 3 ), or an increased CRP on day 3 ( $>45 \mathrm{mg} / \mathrm{l}$ with a stool frequency of 3-8) need to be identified early, as $85 \%$ of these will require colectomy using the criteria in this study. The inadequacies of conventional treatment should be recognised, but the role of cyclosporine for treating severe ulcerative colitis has yet to be defined. After a week of treatment, those patients who have a stool frequency $>3 /$ day or visible blood in the stool have a $60 \%$ chance of continuous symptoms and $40 \%$ chance of colectomy in the months after admission.

We are particularly grateful to Sister and staff on the medical and surgical Gastroenterology wards for their care of the patients, to Dr Helen Chapel and June White, Department of patients, to Dr Helen Chapel and June White, Department of Karen Hayllar, King's College Hospital, London for additional Karen Hayllar, King'
statistical evaluation.

1 Edward FC, Truelove SC. The course and prognosis of ulcerative colitis. Gut 1963; 4: 299-315.

2 Gallagher ND, Goulston SSM, Wyndham N, Morrow W. The management of fulminant ulcerative colitis. Gut 1962; 3: 306-11.

3 Truelove SC, Jewell DP. Intensive intravenous regimen for severe attacks of ulcerative colitis. Lancet 1974; $\mathrm{i}$ 1067-70.

4 Ritchie JK. Results of surgery for inflammatory bowel disease: a further survey of one hospital region. $B M \mathcal{F}$ $1974 ; 1: 264-8$.

5 Truelove SC, Lee EG, Willoughby CP, Kettlewell MGW. Further experience in the treatment of severe attacks of ulcerative colitis. Lancet 1978; ii: 1086-8.

6 Järnerot G, Rolny P, Sandberg-Gertzén H. Intensive intravenous treatment of ulcerative colitis. Gastroenterology venous treatment $1985 ; 89: 1005-13$.

7 Jones HVV, Grogono J, Hoare AM. Acute colitis in a district general hospital. $B M \mathcal{F} 1988 ; 294$ : 683-4.

8 Lichtiger S, Present DH, Kornbluth A, et al. Cyclosporine in severe ulcerative colitis refractory to steroid therapy. N Engl f Med 1994; 330: 1841-5.

9 Lennard-Jones JE, Ritchie JK, Hilder W, Spicer CC. Assessment of severity in colitis: a preliminary study. Gut 1975; 16: 579-84.

10 Buckell NA, Lennard-Jones JE, Hernandez MA, Kohn J, Riches PG, Wadsworth J. Measurement of serum proteins during attacks of ultraviolet as a guide to patient management. Gut 1979; 20: 22-7.

11 Meyers S, Level PK, Feuer EJ, Johnson JW, Janowitz HD. Predicting the outcome of corticoid therapy for acute ulcerative colitis. Results of a prospective randomized ulcerative colitis. Results of a prospective random

12 Chew CN, Nolan DJ, Jewell DP. Small bowel gas in severe ulcerative colitis. Gut 1991; 32: 1535-9.

13 Truelove SC, Witts LJ. Cortisone in ulcerative colitis: preliminary report on a therapeutic trial. $B M \mathcal{F} 1954$; 2 375-8.

14 Jewell DP, Caprilli R, Mortensen N, Nicholls RJ, Wright JP. Indications and timing of surgery for severe ulcerative colitis. Gastroenterology International 1991; 4: 161-4.

15 Jewell DP. Medical management of severe ulcerative colitis. Int $\mathcal{F}$ Colorectal Dis 1988; 3: 186-9.

16 Breiman L, Friedman JH, Olshen RA, Stone CJ. Classification and regression trees. California: Wadsworth, 1984.

17 Rees DC, Satsangi J, Cornelissen PL, Travis SPL, White J, Jewell DP. Are serum concentrations of nitric oxide metabolites useful for predicting the clinical outcome of severe ulcerative colitis. Eur $\mathcal{F}$ Gastroenterol Hepatol 1995; 7: 227-30. 